\title{
HLA-B* 44 Is Associated with Dengue Severity Caused by DENV-3 in a Brazilian Population
}

\author{
Liciana Xavier Eurico de Alencar, ${ }^{1}$ Ulisses de Mendonça Braga-Neto, ${ }^{2}$ \\ Eduardo José Moura do Nascimento, ${ }^{3}$ Marli Tenório Cordeiro, ${ }^{1,4}$ Ana Maria Silva, \\ Carlos Alexandre Antunes de Brito, ${ }^{5}$ Maria da Paz Carvalho da Silva, ${ }^{6}$ \\ Laura Helena Vega Gonzales Gil,, ${ }^{1}$ Silvia Maria Lucena Montenegro, ${ }^{7}$ \\ and Ernesto Torres de Azevedo Marques Júnior ${ }^{1,3}$
}

${ }^{1}$ Virology and Experimental Therapy Laboratory, Aggeu Magalhães Research Center, Fiocruz, Avenida Moraes Rego s/n, Campus da UFPE, Cidade Universitária, 50670-420 Recife, PE, Brazil

${ }^{2}$ Department of Electrical Engineering, Texas A®M University, 3128 TAMU, Station College Station, TX 77843-3128, USA

${ }^{3}$ Department of Infectious Diseases and Microbiology, Center for Vaccine Research, University of Pittsburgh, 3501 Fifth Avenue, Biomedical Science Tower 3, Room 9022, Pittsburgh, PA 15261, USA

${ }^{4}$ Central Laboratory of Public Health, Secretaria de Saúde do Estado de Pernambuco, Praça Oswaldo Cruz s/n, Boa Vista, 50050-210 Recife, PE, Brazil

${ }^{5}$ Hospital das Clínicas, Universidade Federal de Pernambuco, Clínica Médica, Avenida Morais Rego s/n, Campus da UFPE, Cidade Universitária, 50670-420 Recife, PE, Brazil

${ }^{6}$ Departamento de Bioquímica e Biofísica, Centro de Ciências Biológicas, Universidade Federal de Pernambuco, Avenida Moraes Rêgo 1235, Cidade Universitária, 50670-420 Recife, PE, Brazil

${ }^{7}$ Departamento de Imunologia do Centro de Pesquisas Aggeu Magalhães-CPqAM, Fiocruz, Avenida Moraes Rego s $/ n$, Campus da UFPE, Cidade Universitária, 50670-420 Recife, PE, Brazil

Correspondence should be addressed to Ernesto Torres de Azevedo Marques Júnior; marques@pitt.edu

Received 31 January 2013; Revised 11 April 2013; Accepted 15 April 2013

Academic Editor: Jean-Paul Gonzalez

Copyright (C) 2013 Liciana Xavier Eurico de Alencar et al. This is an open access article distributed under the Creative Commons Attribution License, which permits unrestricted use, distribution, and reproduction in any medium, provided the original work is properly cited.

\begin{abstract}
Human leukocyte antigen (HLA) alleles have been correlated with susceptibility or resistance to severe dengue; however, few immunogenetic studies have been performed in Latin American (LA) populations. We have conducted immunogenetic studies of HLA class I and II alleles in a cohort of 187 patients with DENV-3 infection and confirmed clinical diagnosis of either severe dengue, known as dengue hemorrhagic fever (DHF), or the less severe form, dengue fever (DF), in Recife, Pernambuco, Brazil. An association analysis was performed using Fisher's association test, with odds ratios (ORs) calculated using conditional maximum likelihood estimates. HLA-B ${ }^{*} 44(P=0.047, \mathrm{OR}=2.025,95 \% \mathrm{CI}=0.97-4.24)$ was found to be associated with increased susceptibility to DHF in response to DENV-3 infection. In addition, HLA-B 07 ( $P=0.048, \mathrm{OR}=0.501$, one-sided $95 \% \mathrm{CI}=0$ $0.99)$ and HLA-DR ${ }^{*} 13(P=0.028, \mathrm{OR}=0.511$, one-sided $95 \% \mathrm{CI}=0-0.91)$ were found to be associated with resistance to secondary dengue infection by DENV-3. These results suggest that HLA-B* 44 supertype alleles and their respective T-cell responses might be involved in susceptibility to severe dengue infections, whereas the HLA-B* 07 supertype alleles and $\mathrm{DR}^{*} 13$ might be involved in cross-dengue serotype immunity.
\end{abstract}

\section{Introduction}

Dengue virus (DENV) has four serotypes, named as DENV-1, DENV-2, DENV-3 and DENV-4. The majority of dengue infections are subclinical; however, the clinical manifestations of dengue infection range from the benign, self-limited dengue fever (DF) to a vasculopathy syndrome known as dengue hemorrhagic fever (DHF) that can lead 
to hypovolemic dengue shock syndrome (DSS). It is often observed in epidemiological studies that the more severe illness occurs more frequently in secondary heterotypic dengue infection [1-7]. There are two main hypotheses to explain the higher frequency of DHF in secondary infections. The first is that heterotypic antibodies would bind to the dengue virus and would facilitate the viral entry into cells expressing Fc receptors; this theory is known as antibody dependent enhancement (ADE) $[8,9]$. The second hypothesis is that anti-dengue memory $\mathrm{T}$ cells that cross-react with related but altered peptide epitopes would induce the $\mathrm{T}$ cells to produce abnormal levels of cytokines leading to vasculopathy. This theory is known as "original antigenic sin" (OAS) or "altered peptide ligand" (APL). The theories are not mutually exclusive, and both are based on the fact that previous dengue infection is a risk factor for developing more severe disease. However, $99 \%$ or more of the secondary dengue infections are benign, suggesting that host and virological genetic factors may be also involved.

The geographic distribution of the DENV and the incidence of the viral infection have grown fast in recent decades, and dengue fever is considered to be one of the most important reemergent tropical diseases [10]. However, there are significant differences in the clinical and epidemiological profile of dengue between the countries of Latin America (LA) and Southeast Asia (SA). In SA countries DHF cases are most common and the dengue morbidity and mortality are more prevalent in children under 15 years of age, whereas in LA countries most of the dengue cases are mild and manifest mostly in adults [11-14]. The differences in the age distribution between LA and SA can be attributed to the differences on the history of these epidemics in these regions and their age profiles are likely to converge over time [15]. However, the differences on the frequency of severe cases may be also partially explained by genetic differences between these populations.

It has been proposed that a high prevalence of dengue resistance genes in the LA population could explain the differences on the frequency of severe cases between SA and LA $[16,17]$. Host genetic polymorphisms involved in innate immune responses have been shown to be correlated with resistance to DHF, such as a variant of the FcGRIIA allele [18], functional polymorphisms of MBL2 [19], and the polymorphism of the CD209 promoter [20]. Similarly, studies of MHC-encoded transporters associated with antigen processing (TAP) genes have also shown associations with DHF $[21,22]$. In addition, the analyses of tumor necrosis factor (TNF) and lymphotoxin alpha (LTA) genes have recently revealed specific combinations of TNF, LTA, and HLA class I alleles that associate with DHF and production of LTA and TNF [23].

Indeed, several aspects of $\mathrm{T}$ cell functionality are altered in DHF patients, including proliferation, activation status, production of cytokines, and their survival [4, 24-26]. All these functions are influenced by specific recognition, through $\mathrm{T}$ cell receptors (TCRs), of the antigen associated with HLA molecules. Thus, polymorphisms of HLA genes may also play an important role in dengue severity. Several genetic variations in HLA class I alleles have been found to correlate with dengue severity in Southeast Asian populations. For example, a protective role for HLA-A*33 in the ethnic Vietnamese [27] and HLA-A*0203 in the ethnic Thai population has been reported [28], as has a pathogenic role for HLA-A* 24 in the Vietnamese [27] and HLA-A* 0207 [28] and HLA-B* 13 [29] in the Thai population. However, there have been fewer studies examining possible correlations between dengue severity and HLA variation in LA populations. In Mexico, LaFleur and colleagues (2002) reported that $\mathrm{HLA}-\mathrm{DRB1}{ }^{*} 08$ and $\mathrm{DRB1}^{*} 04$ were associated with susceptibility and resistance, respectively, to developing DHF [30]. HLA-A* 1 and Cw1 were associated with shock, dehydration, and severe hemorrhage (DHF/dengue shock syndrome) in the Cuban population [31].

Eighty percent of dengue cases reported in LA occur in Brazil, but studies correlating dengue severity and HLA alleles in this country are scarce. In order to determine if the Brazilian HLA allelic diversity plays a role in protection or susceptibility to DHF, we have analyzed the HLA-A, B, C, $\mathrm{DR}$, and DQ genotypes of a cohort of dengue patients from Recife, Brazil. We found that patients bearing the HLA-B ${ }^{*} 44$ allele had increased susceptibility to DHF. We also found that the alleles HLA-B* 50 and $-\mathrm{DR}^{*} 16$ were associated with increased risk for DHF, whereas HLA-DR* 9 and -DR* 12 were associated with protection against $\mathrm{DHF}$, but these alleles were not sufficiently prevalent in the population (allelic frequency $<5 \%$ ) for us to reach a significant conclusion. However, the allele HLA-B*50 is part of the HLA-B* 44 supertype, lending support to the hypothesis that HLA-B*50 is in fact a risk factor for DHF. In addition, we found that individuals with the HLA-B ${ }^{*} 07$ and HLA-DR* 13 alleles appeared to be resistant to secondary infection by DENV-3.

\section{Methods}

2.1. Ethics Statement. This study was reviewed and approved by the Ethics Committee of the Brazilian Ministry of Health CONEP: 4909; Process number 25000.119007/2002-03; CEP 68/02. FWA00001515; IRB00001858. In addition, the Johns Hopkins University Institutional Review Board also reviewed and approved this study as protocol JHM-IRB-3: 03-08-2701. Informed consent was obtained from all volunteers and all clinical investigation was conducted according to the principles expressed in the Declaration of Helsinki.

2.2. Study Design. A cohort of dengue patients admitted to hospitals in the city of Recife, state of Pernambuco, Brazil, was characterized. During this study there was a large outbreak of DENV-3 and most of our volunteers were of this serotype, so for this study we selected only individuals infected with DENV-3. This cohort has been characterized previously in $[32,33]$. Briefly, multiple blood samples collected during the first 30 days after the appearance of the symptoms were used to obtain sera, plasma, and peripheral blood mononuclear cells (PBMC). All specimens were cryopreserved for further characterization of the cohort. Dengue diagnosis was performed using a combination of virus isolation in C6/36 cells [34] and RT-PCR [35] of samples collected on the day 
of admission (first sample), together with ELISAs to detect antidengue IgM and IgG in the first and follow-up samples.

Dengue cases were confirmed by ELISA, RT-PCR, or virus isolation and were clinically classified according to the classic World Health Organization (WHO) criteria into two classes: dengue fever (DF) and dengue hemorrhagic fever (DHF) [36]. DF cases were characterized by high fever lasting for 7 days and accompanied by at least two of the following symptoms: severe headache, retroorbital pain, myalgia, arthralgia, and rash associated with platelet counts above $100,000 / \mathrm{mm}^{3}$. Dengue hemorrhagic fever cases were defined as having the same clinical manifestations as for DF, but with evidence of hemorrhage, thrombocytopenia (platelets $<100,000 / \mathrm{mm}^{3}$ ), and plasma leakage following defervescence. The classification of primary and secondary dengue infections was based on the kinetics of the IgM and IgG response, according to validated criteria published previously [32]: primary infection $(\mathrm{P})$ was defined as the absence of specific antidengue IgG antibodies in the first serum samples during the acute phase, with IgM, virus, and/or viral RNA being detected and followed by the presence of antidengue IgG in convalescence serum samples. Secondary infection (S) was defined as the detection of specific antidengue IgG in the first acute sample and the absence of antidengue IgM, associated with a positive RT-PCR and/or virus isolation. With the exception of a few cases, antidengue IgM was present in the convalescent serum samples.

2.3. DNA Extraction. Genomic DNA was extracted from frozen PBMC samples using the PureLink Genomic DNA (Invitrogen) kit, according to the manufacturer's instructions. The cells were digested with Proteinase $\mathrm{K}$ at $55^{\circ} \mathrm{C}$ using an optimized digestion buffer formulation that aids in protein denaturation and enhances Proteinase K activity. Any residual RNA was removed by digestion with RNAse A prior to binding samples to the silica membrane. The lysate was mixed with ethanol and PureLink Genomic Binding Buffer that allowed high DNA binding PureLink Spin Column. The DNA bound to the silica-based membrane in the column and impurities were removed by thorough washing with Wash Buffers. The genomic DNA was then eluted in low salt Elution Buffer. The isolated DNA was $20-50 \mathrm{~kb}$ in size and was suitable for PCR. The samples were stored at $-20^{\circ} \mathrm{C}$ and later used for HLA typing.

2.4. HLA Typing. Extracted DNA was used for lowresolution HLA typing carried out by PCR with Sequence Specific Primers amplification methods (Commercial kit Invitrogen SSP UniTray) as previously described $[37,38]$. Setup included mixing a reaction buffer with human genomic DNA sample and Taq DNA Polymerase, dispensing the mixture to the UniTray sealing and then performing thermal cycling. After cycling was completed the PCR products were loaded onto a $2 \%(\mathrm{w} / \mathrm{v})$ agarose gel for electrophoresis. Next the gel was stained with ethidium bromide, photographed using a gel documentation system UV transilluminator, and analyzed with UniMatch Plus software in order to determine the HLA-A, HLA-B, HLA-Cw, HLA-DR, and HLA-DQ loci using a worksheet for the specific amplification patterns.

2.5. Statistical Analysis. In order to analyze the potential associations between different groups (primary versus secondary infections; DF versus DHF), we determined the frequency of a given allele in each of the groups under analysis. We then applied the two-sided Fisher's test on each allele, in order to verify the possibility of significant differences between the frequencies of DF versus DHF. For the comparison between frequencies of primary and secondary infections we employed a one-sided Fisher's test for $\mathrm{OR}<1$, as the only effect of interest (protection against secondary infection). The reported OR and associated confidence interval limits in both cases are estimated using the conditional maximum-likelihood (CML) method. No correction for multiple testing was attempted, implying that further validation of the results with additional patients in follow-up studies is recommended. In this regard, significant alleles with an allelic frequency inferior to $5 \%$ need to be appreciated with caution.

\section{Results}

3.1. Validation of the Genotyping Data. The HLA type of the HLA-A, HLA-B, HLA-C, HLA-DR, and HLA-DQ loci of the 187 confirmed dengue of DENV-3 genotype III [32] was determined in low resolution. The summary of the demographic profile of the patient volunteers in this study is shown on Table 1 and the complete table is shown in supplemental material Table S1 (see Supplementary Materials available online at http://dx.doi.org/10.1155/2013/648475).

The quality and representativeness of the HLA genotyping data of our dengue cohort were initially accessed by comparing the frequency of the alleles identified to those found in two samples of Brazilian populations of normal blood bank volunteers deposited in the PubMed's MHC database (dbMHC) (http://www.ncbi.nlm.nih.gov/mhc/): one population was from the state of Minas Gerais (designated here as data set $\mathrm{dbMHCl}$ ) and another from the state of São Paulo (designated dbMHC2). The dbMHC1 data set contains only HLA-A and HLA-B genotypes, whereas the dbMHC2 dataset contains HLA-A, HLA-B, HLA-C, and HLA-DR genotypes. These databases are considered to represent the HLA diversity of these populations $[39,40]$. High-resolution genotyping information in the dbMHC data sets was ignored, and alleles that were not carried by any of the patients were also ignored, so as not to artificially inflate the correlation. In Table 2 it is presented the results with the correlation coefficients, confidence intervals, and significance. These analyses indicated that there was a high correlation between the observed allelic frequencies in our cohort named LaViTE and both dbMHCl and dbMHC2 data sets indicating that our sample does not have significant differences on the HLA profile from these other two Brazilian groups. This result indicates that the genotype of the population making up our cohort is representative of the Brazilian genetic background for all the available MHC 
TABLE 1: Distribution of DENV-3 cases with regard to demographic profiles, clinical manifestation, and type of infection.

\begin{tabular}{lccccccr}
\hline Clinical diagnosis & \multirow{2}{*}{ Median age (range) } & \multicolumn{2}{c}{ Sex } & \multirow{2}{*}{ IgM (+) } & IgG (+) & \multicolumn{2}{c}{ Type of infection } \\
Primary & Secondary & Total \\
\hline DF & $27(4-76)$ & 61 & 59 & 79 & 75 & 47 & 73 \\
DHF & $17(0,6-84)$ & 25 & 42 & 53 & 48 & 19 & 48 \\
\hline
\end{tabular}

DF: dengue fever; DHF: dengue hemorrhagic fever; IgM: immunoglobulin M; IgG: immunoglobulin G.

TABLE 2: Correlations between the observed allelic frequencies in the LaViTE cohort and two other Brazilian datasets deposited in the dbMHC database of the NCBI, dbMHC1 (Minas Gerais, Brazil), and dbMHC2 (São Paulo, Brazil).

\begin{tabular}{lccc}
\hline HLA loci & Correlation & $95 \%$ CI & $P$ value \\
\hline HLA-A & & & \\
LaViTE, dbMHC1 & 0.9776 & $0.9415-0.9915$ & $<10^{-12}$ \\
LaViTE, dbMHC2 & 0.9445 & $0.8588-0.9788$ & $<10^{-8}$ \\
HLA-B & & & \\
LaViTE, dbMHC1 & 0.9171 & $0.8357-0.9591$ & $<10^{-12}$ \\
LaViTE, dbMHC2 & 0.8223 & $0.6640-0.9100$ & $<10^{-8}$ \\
HLA-C & & & \\
LaViTE, dbMHC2 & 0.9114 & $0.7374-0.9719$ & $<10^{-5}$ \\
HLA-DR & & & \\
LaViTE, dbMHC2 & 0.7977 & $0.4401-0.9368$ & $<0.05$ \\
\hline
\end{tabular}

HLA: human leukocyte antigen; CI: confidence interval.

TABLE 3: Results of one-sided Fisher's test $(\mathrm{OR}<1)$ of association between the various HLA alleles analyzed and the type of dengue infection (primary versus secondary infections).

\begin{tabular}{lcccc}
\hline \multirow{2}{*}{ HLA allele } & \multicolumn{2}{c}{ Frequency $(\%)$} & \multicolumn{2}{c}{ Fisher's test } \\
& Primary & Secondary & OR $(95 \% \mathrm{CI})$ & $P$ \\
\hline B07 & 13.33 & 7.14 & $0.501(0-0.99)$ & 0.048 \\
DR13 & 18.64 & 10.45 & $0.511(0-0.91)$ & 0.028 \\
\hline
\end{tabular}

HLA: human leukocyte antigen; OR: odds ratio; CI: confidence interval; $P$ : $P$ value.

genes investigated here. The relative frequencies of the alleles studied in the various data sets are shown in Figure 1.

3.2. Association between HLA Allele and Dengue Infection History. It is postulated that CD4 and CD8 T cell responses play a role in protection against flavivirus and this has been shown in epidemiological studies [41] and in animal models [42], although the requirements for $\mathrm{T}$ cell mediated protection is not known. HLA alleles can bind to different peptide repertoires and some are more permissive to a wider range of peptides than others [43]. In addition, some of the peptides epitopes can be more conserved across serotypes than others. It is also known that dengue infection by one serotype leads to cross-serotype immunity that can last for several months and this protection is thought to be mediated by $\mathrm{T}$ cells $[44,45]$. Based on this, we postulated that some HLA alleles would be more capable of producing cross-serotype $\mathrm{T}$ cell responses and if so the presence of this allele could associate to reduced susceptibility to secondary infections. To test this hypothesis we investigated the associations between dengue infection history (primary $\mathrm{X}$ secondary) and the HLA allelic frequency. Sixty-six (35.3\%) of the cases were diagnosed as having primary infections ("Prim," Table 1) and $121(64.7 \%)$ as having secondary infections ("Sec," Table 1). A one-sided Fisher's association test was used to identify differences in the distribution of each allele of a given HLA haplotype with regard to the type of infection, whether primary or secondary (Table 3). The one-sided test seeks to identify OR $<1$, as the only effect of interest in this case (protection against secondary infection). According to this association test, two alleles, HLA-B*07 $(P=0.048$, $\mathrm{OR}=0.501$, one-sided $95 \% \mathrm{CI}=0-0.99$ and HLA-DR ${ }^{*} 13$ $(P=0.028$, OR $=0.511$, one-sided 95\% CI $=0-0.91)$, were found to be significantly associated at the 95\% level. Among patients with the HLA-B* 07 genotype, $13.33 \%$ were characterized as having primary infection, whereas $7.14 \%$ had a secondary infection, suggesting that this allele might be involved in eliciting cross-serotype protective $\mathrm{CD}^{+} \mathrm{T}$ cell responses. Similarly, among dengue patients with the HLA-DR ${ }^{*} 13$ genotype, $18.64 \%$ were characterized as having primary infection, whereas $10.45 \%$ had a secondary infection, suggesting that this allele is also likely to elicit cross-protective responses against heterotypic dengue serotypes. Figure 2 shows comparison of the frequency of all the alleles studied, with regard to the type of infection. Although these results are statistically significant these associations need to be further validated in larger cohorts.

\subsection{Association between HLA Haplotype and Dengue Severity.} $\mathrm{T}$ cell responses have been associated with both protection and also severity of the disease [46-48] and several HLA alleles have been associated with protection and susceptibility to severe disease [27-29]. So, we next examined the potential association between HLA allele and clinical outcome in 120 (64\%) DF patients and 67 (36\%) DHF patients. Taking the same approach that we had used for our analysis of the type of infection, we used Fisher's test to determine the difference in the distribution of each allele of a given HLA allele between DF and DHF patients (Table 4). The alleles that showed significant association with increased risk for DHF at the $95 \%$ level were $\mathrm{B}^{*} 44(P=0.047, \mathrm{OR}=2.025,95 \% \mathrm{CI}=0.97-$ 4.24), $\mathrm{B}^{*} 50(P=0.037, \mathrm{OR}=4.542,95 \% \mathrm{CI}=1.01-27.72)$, and $\mathrm{DR}^{*} 16(P=0.046, \mathrm{OR}=3.173,95 \% \mathrm{CI}=0.93-12.34)$. In addition, the alleles $\mathrm{DR}^{*} 09$ and $\mathrm{DR}^{*} 12$ (both with $P=0.049$, $\mathrm{OR}=0,95 \% \mathrm{CI}=0-1.15)$ appeared to have a protective effect against DHF. However, the alleles $\mathrm{B}^{*} 50, \mathrm{DR}^{*} 16$, and especially $\mathrm{DR}^{*} 9$ and $\mathrm{DR}^{*} 12$ were all rare in the studied population, with an overall allelic frequency of less than $5 \%$, indicating that the results for these alleles need to be appreciated with 


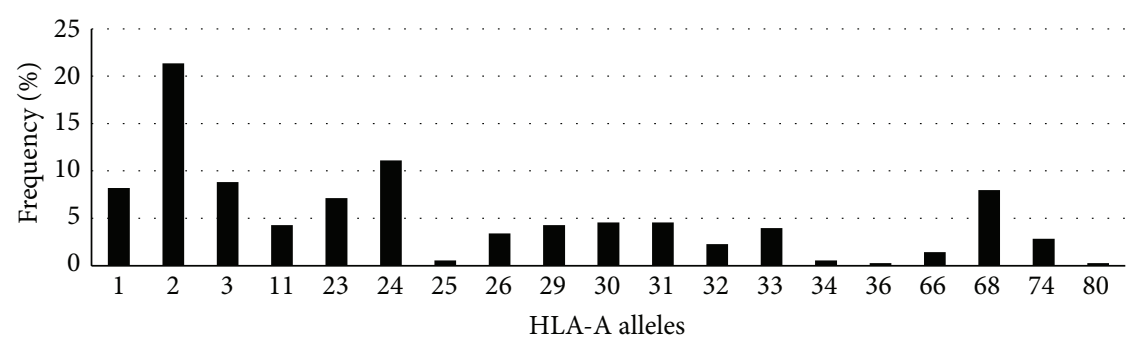

(a)

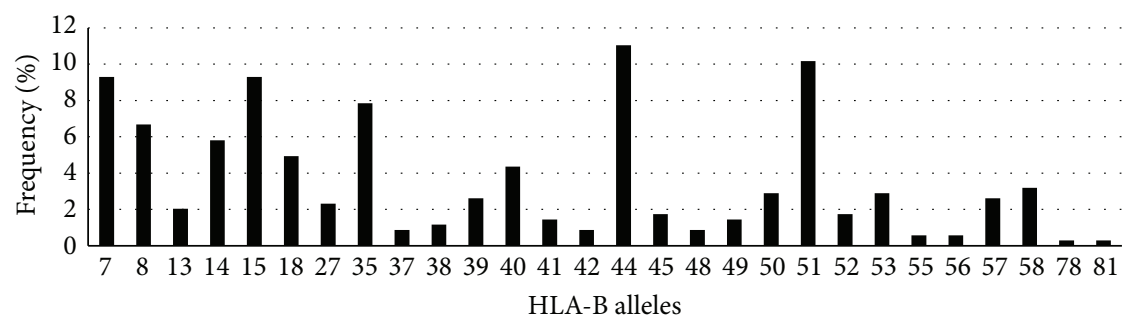

(b)

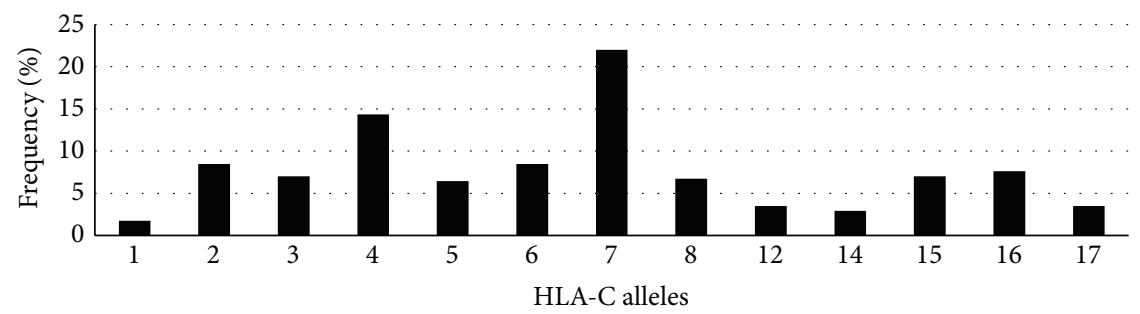

(c)

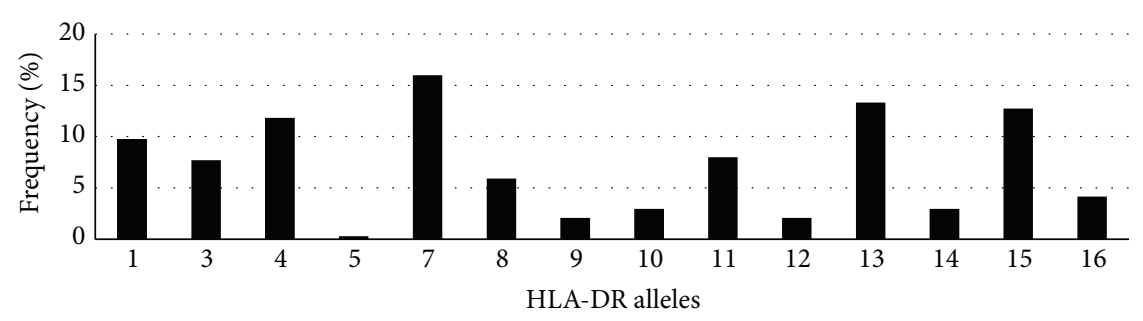

(d)

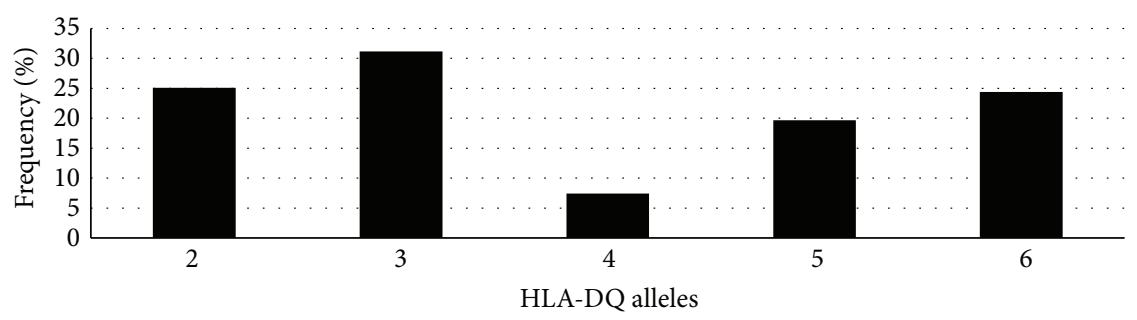

(e)

FIgUre 1: General frequency for HLA in the study population. (a) HLA-A, (b) HLA-B, (c) HLA-C, (d) HLA-DR, and (e) HLA-DQ.

caution. The $\mathrm{B}^{*} 44$ allele is a very common HLA-B allele in our study population ( $11 \%$ frequency) and, according to the results, its frequency is nearly twice as high in DHF patients $(15.83 \%)$ as in DF patients (8.48\%, Figure 3), reason why confidence is increased that this result is a true positive association. The allele $B^{*} 50$ was reported in $5.83 \%$ of the DHF cases and only in $1.34 \%$ of the DF cases. HLA-B ${ }^{*} 50$ encodes a protein that has a similar peptide binding profile as the HLA-B ${ }^{*} 44$, being considered a member of the B44 supertype; this lends support to the hypothesis that the repertoire of $\mathrm{T}$ cell responses elicited by the $\mathrm{B}^{*} 44$ and $\mathrm{B}^{*} 50$ alleles may be involved in the DHF pathogenic mechanism. Allele DR* ${ }^{*} 16$ was reported in $7.14 \%$ of the DHF cases and only in $2.36 \%$ of the DF cases, also suggesting an increased susceptibility 


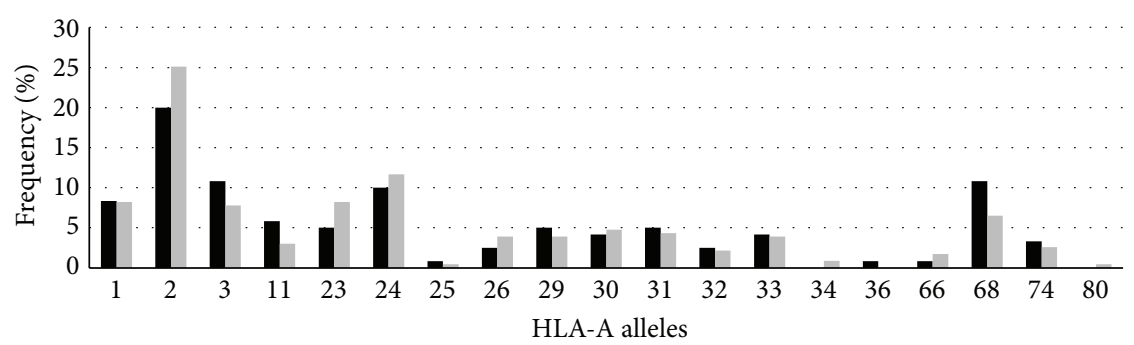

(a)

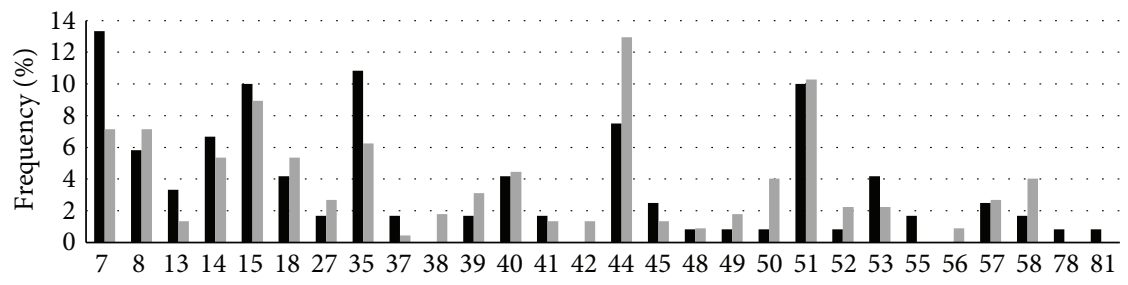

HLA-B alleles

(b)

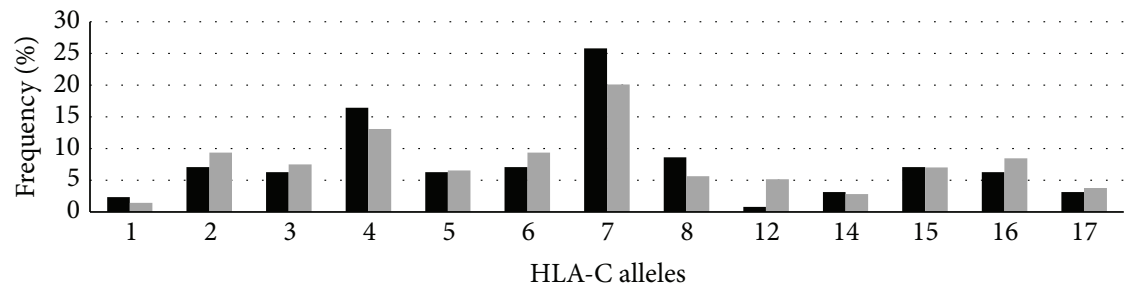

(c)

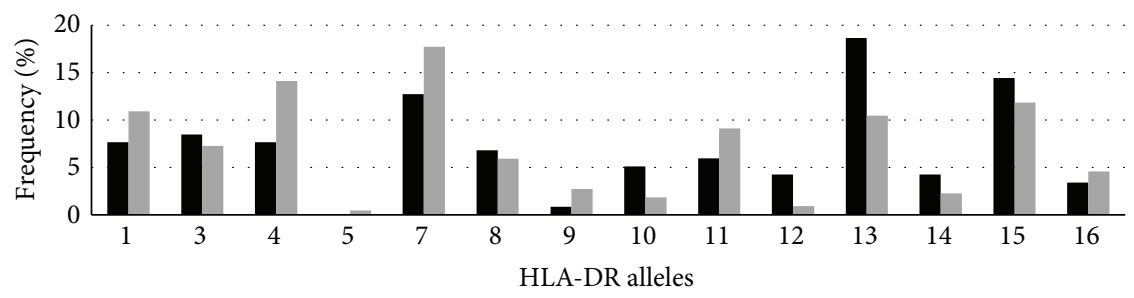

(d)

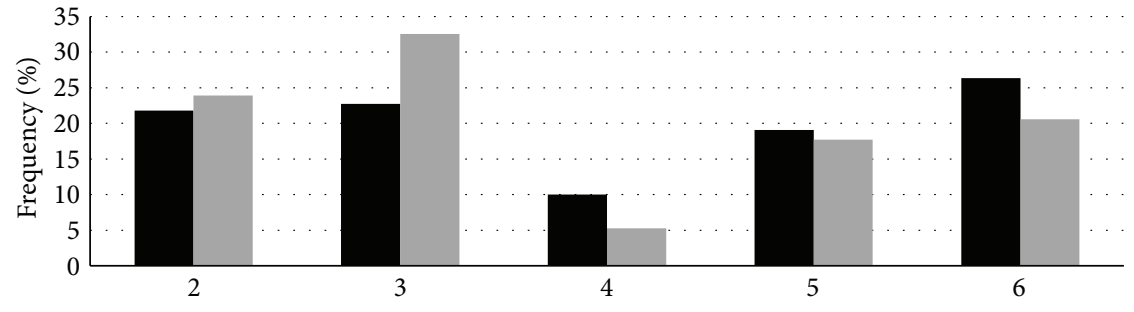

HLA-DQ alleles

- Primary

- Secondary

(e)

Figure 2: Allele versus primary-secondary frequency for HLA in the study population. (a) HLA-A, (b) HLA-B, (c) HLA-C, (d) HLA-DR, and (e) HLA-DQ

to DHF. By contrast, the HLA-DR* 9 and $\mathrm{DR}^{*} 12$ genotypes were found to be protective; both had a $3.3 \%$ frequency in DF patients, whereas no DHF patients had the $\mathrm{DR}^{*} 9$ and $\mathrm{DR}^{*} 12$ genotypes.

\section{Discussion}

In this study we have genotyped HLA-A, HLA-B, HLA-Cw, HLA-DR, and HLA-DQ loci of 187 well-characterized dengue 
TABLE 4: Results of two-sided Fisher's test of association between the HLA alleles analyzed and the clinical manifestations of dengue (DHF versus DF).

\begin{tabular}{lcccc}
\hline \multirow{2}{*}{ HLA allele } & \multicolumn{2}{c}{ Frequency (\%) } & \multicolumn{2}{c}{ Fisher's test } \\
& DF & DHF & OR (95\% CI) & $P$ \\
\hline B44 & 8.48 & 15.83 & $2.02(0.97-4.24)$ & 0.047 \\
B50* $^{*}$ & 1.34 & 5.83 & $4.54(1.01-27.72)$ & 0.037 \\
DR9 $^{*}$ & 3.3 & 0 & $0(0-1.15)$ & 0.049 \\
DR12 $^{*}$ & 3.3 & 0 & $0(0-1.15)$ & 0.049 \\
DR16 $^{*}$ & 2.36 & 7.14 & $3.17(0.93-12.34)$ & 0.046 \\
\hline
\end{tabular}

HLA: human leukocyte antigen; DF: dengue fever; DHF: dengue hemorrhagic fever; OR: odds ratio; CI: confidence interval; $P$ : $P$ value.

Alleles with overall allelic frequency inferior to $5 \%$ are indicated with a star.

patients and this is the largest immunogenetic study performed in LA population reported to date. The analysis identified associations of HLA-B ${ }^{*} 44,-B^{*} 50$, and $-\mathrm{DR}^{*} 16$ alleles with susceptibility to DHF, whereas HLA-DRB1*09 and $-\mathrm{DR}^{*} 12$ were associated with protection to DHF. In addition, the HLA-B ${ }^{*} 07$ and $-\mathrm{DR}^{*} 13$ were associated with lower frequency of secondary dengue infections suggesting that these HLA alleles might be involved in presenting protective cross-reactive $\mathrm{T}$ cell epitopes.

Dengue $\mathrm{T}$ cell responses have been correlated with dengue protection and pathogenesis and are considered as a double-edged sword. Immunological studies have shown that DHF patients present decrease in $\mathrm{T}$ cell proliferation and IFN $\gamma$ production before defervescence [24]. In addition, increased levels of activated T cells [49], expressing cytokines [25] and undergoing apoptosis, are also observed during the acute phase of dengue infection $[4,26]$. All of these functions are thought to be dependent upon specific recognition, through $\mathrm{T}$ cell receptors (TCRs), of particular antigens associated with HLA molecules. Indeed, $\mathrm{CD} 8^{+} \mathrm{T}$ cells-specific response against a dengue NS3 epitope has been associated with disease severity; however, the mechanisms of specific $\mathrm{T}$ cell responses on pathogenesis are not understood [46]. Several studies have shown statistically significant associations between dengue disease severity and specific HLA molecules [50]. Thus, HLA peptide epitope repertoire may be a factor determining patient outcomes in the case of dengue infection either by eliciting protective $T$ cell responses against cross-serotype conserved sequences [51] or by eliciting T cell responses to variable epitopes that can lead to APL or OAS responses $[4,52]$.

Four immunogenetic studies have explored HLA association with dengue severity in LA populations $[30,31,53,54]$. Paradoa Pérez et al. have found that $\mathrm{A}^{*} 01$ was associated to susceptibility DHF, whereas $A^{*} 29$ was associated with resistance in a cohort of 82 patients [31] in Cuba. Sierra and colleagues (2007) studied a cohort of 120 dengue patients and found that $\mathrm{A}^{*} 31$ and $\mathrm{B}^{*} 15$ were associated with susceptibility to DHF and DRB1*04 and DRB1 ${ }^{*} 07$ were associated with resistance to DHF [54]. Consistent with Sierra study, LaFleur et al. also found that $\mathrm{DRB1}^{*} 04$ was associated with resistance to DHF in a Mexican cohort of 47 patients [30]. In our study we did not find an association between $\mathrm{A}^{*} 01$ (Figure 3 ) and DHF, and we found a slightly lower frequency of $A^{*} 29$ and
$\mathrm{A}^{*} 31$ in DHF patients; these alleles had a very low frequency in our cohort and the frequencies were not statistically significant in DF from DHF. However, we observed a larger frequency of $B^{*} 15$ in DHF, but it was not significant. We did not observe any protective effect of DRB1* 04 or ${ }^{*} 07$ in our cohort. However, here are many methodological differences among all these studies to allow a comprehensive comparison and these differences together with other environmental and epidemiological differences can explain the different findings in the different studies. To date, there have been no reports regarding potential correlations between dengue severity and HLA alleles in Brazil. In the only study performed in this context in a Brazilian population, the authors compared polymorphisms in HLA classes I and II in DF patients and healthy people, associating HLA-DQ1 with susceptibility to dengue infection [53].

Similar immunogenetic studies performed have been performed in SA. However, the size of the study cohorts was in general significantly larger than the ones in LA. Chiewsilp et al. [29] found that $A^{*} 02$ was associated with susceptibility to DHF, whereas $\mathrm{B}^{*} 13$ was found associated with resistance. In a larger study in a Thai population of 263 dengue patients, Stephens and colleagues (2002) [28] also found an association with $\mathrm{A}^{*} 02$; however, in this study they used high resolution HLA genotyping and identified the association to be with the $\mathrm{A}^{*} 0207$ subgenotype and was specific for DENV-2. In addition, they also found that HLA $A^{*} 11, B^{*} 51$, and $B^{*} 46$ were associated with susceptibility to DHF, whereas the HLA B* 15 and $B^{*} 44$ were associated with resistance to secondary DHF infections. In fact, the same HLA $B^{*} 44$ was associated with increased risk in patients with primary DHF infections in Thai population. The $\mathrm{B}^{*} 44$ association with resistance to DHF in secondary infections and susceptibility to DHF in primary infection is intriguing. It suggests that studies about the role of HLA alleles in dengue disease need to take in consideration the serotype and the order of infection. In subsequent study and in a larger Thai group of 435 dengue patients Vejbaesya and colleagues (2009) [23] found that HLA B* 48 was associated with DHF, however the previous associations found were not confirmed. In a Vietnamese study with 352 dengue patients Loke and colleagues (2001) [27] identified correlations between HLA A* 24 and susceptibility to DHF grades III and IV and correlation between $A^{*} 33$ and resistance to DHF grades III and IV. In other Vietnamese study [55] was reported HLA association between the HLA A* 24 allele variant with a histidine at position 70 and dengue shock in primary infections, whereas the HLA DRB1*0901 was found associated with resistance to dengue shock syndrome as result of DV2. The results presented here are consistent with the associations found by Nguyen and colleagues (2008) [55]; we also found that the $\mathrm{DRB1}^{*} 09$ is associated with protection to DHF. The results of our study were also consistent with the ones of Chiewsilp and colleagues (1981) [29]; there was a slightly higher frequency of $\mathrm{A}^{*} 02$ in DHF patients as compared to DF and we also observed that the $\mathrm{B}^{*} 13$ allele was more frequent in $\mathrm{DF}$ than in DHF; however, the frequency of the $\mathrm{B}^{*} 13$ allele in our cohort is low and neither of these differences was statistically significant. As the study of Stephens and colleagues (2002) 


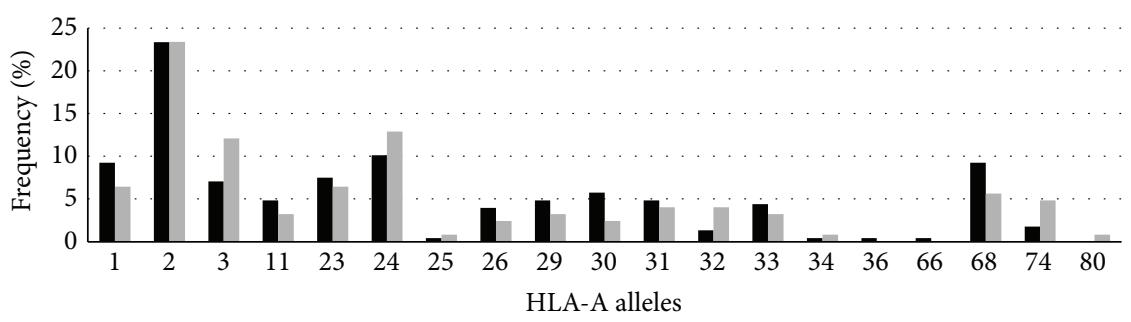

(a)

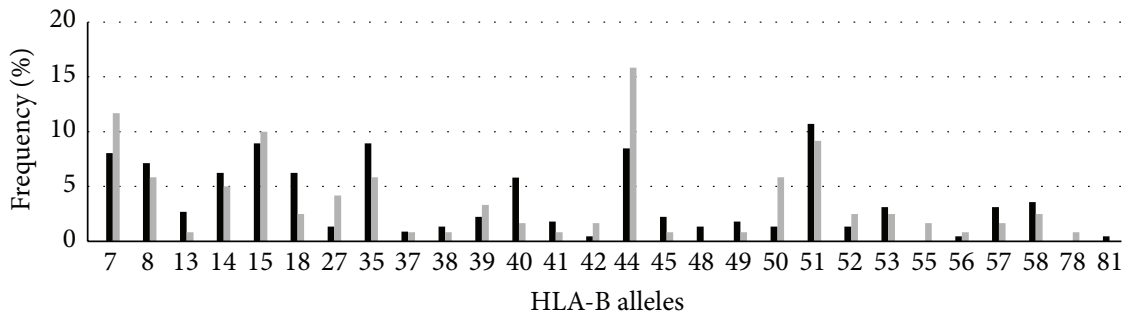

(b)

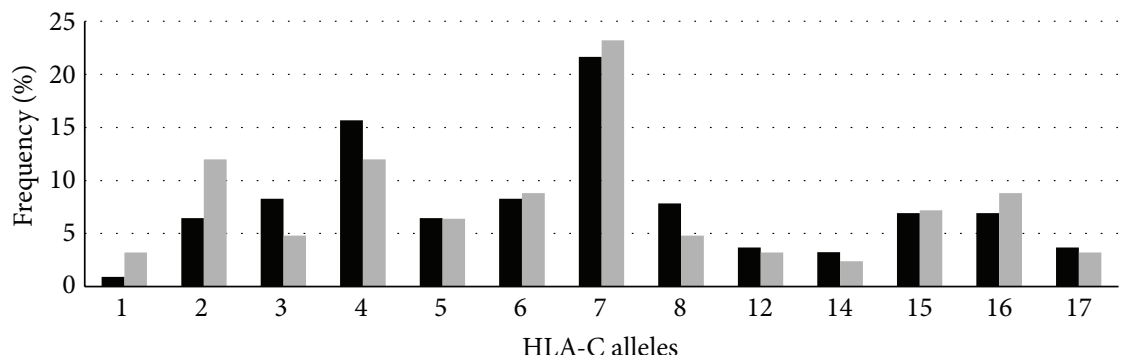

(c)

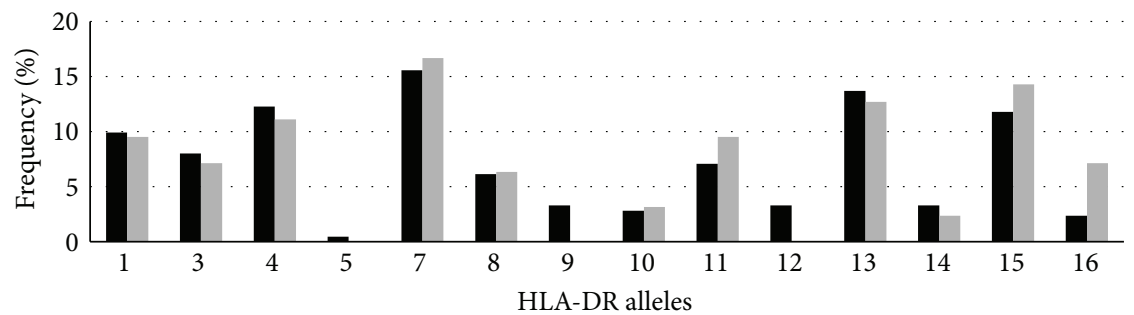

(d)

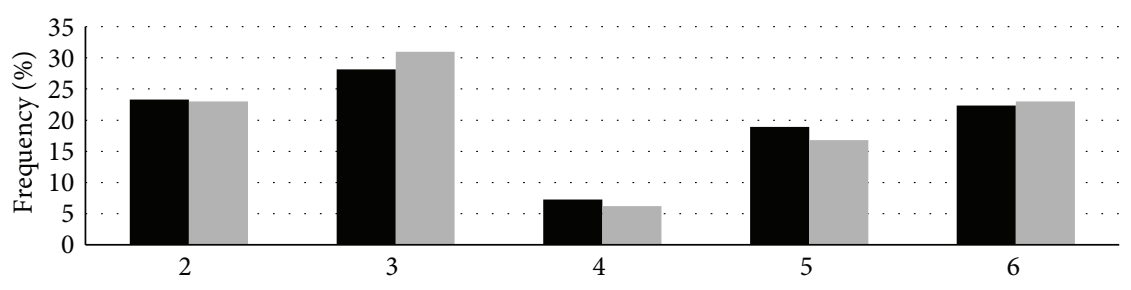

HLA-DQ alleles

$$
\text { - DF }
$$

DHF

(e)

FIGURE 3: Allele versus DF-DHF frequency for HLA in the study population. (a) HLA-A, (b) HLA-B, (c) HLA-C, (d) HLA-DR, and (e) HLA-DQ. DF: dengue fever; DHF: dengue hemorrhagic fever. 
[28], we found highly significant associations between $B^{*} 44$ with the severity of the dengue disease. In our study, the allele $B^{*} 44$ was associated with susceptibility to DHF and in the associations reported by Stephens the $B^{*} 44$ showed susceptibility to DHF only in primary infections. In a more recent and very elegant study performed in a Sri Lankan cohort of 110 DHF patients, and a control group of 119 individuals who had never reported a dengue fever was found that HLA-A 31 and $\mathrm{DRB1}^{*} 08$ were associated with susceptibility to DHF [56]. In our study we did not find differences in the HLA frequencies of our dengue cohort and the general population. However in our cohort, we only have DF and DHF grades I and II, and our control population is random and does not allow a similar type of comparison.

In general, several HLA alleles have been associated with resistance and susceptibility to DHF; however, the results are not consistent and still lack a proper demonstration of cause and effect. Of course, it is clear that multiple factors are involved in the pathogenesis of DHF. The findings presented here show a significant association of the HLA- $\mathrm{B}^{*} 44,-\mathrm{B}^{*} 50$, and $-\mathrm{DR}^{*} 16$ alleles with increased susceptibility to DHF and represent another attempt to improve our understanding of the role of HLA molecules on outcome of DENV infection. However, it is important to perform additional genetic studies in larger dengue cohorts.

\section{Acknowledgments}

The authors would like to acknowledge the support of the National Institute of Allergy and Infectious Diseases (NIAID/NIH) through Grant U19 AI56541 and additional support from the Brazilian National Research Council (CNPq 150911/2003-3 to EJMN), PAPES V (403652/2008-1) and PDTIS RVR09-FIOCRUZ. We also thank all the staff of the Hospital Esperança, Hospital Santa Joana, and Instituto Materno Infantil de Pernambuco (IMIP), Recife, for their valuable cooperation in this project. Silvia M.L. Montenegro is a recipient of $\mathrm{CNPq}$ fellowships.

\section{References}

[1] S. B. Halstead, "Pathogenisis of dengue: challenges to molecular biology," Science, vol. 239, no. 4839, pp. 476-481, 1988.

[2] M. M. Mangada, T. P. Endy, A. Nisalak et al., "Denguespecific $\mathrm{T}$ cell responses in peripheral blood mononuclear cells obtained prior to secondary dengue virus infections in Thai schoolchildren," Journal of Infectious Diseases, vol. 185, no. 12, pp. 1697-1703, 2002.

[3] M. M. Mangada and A. L. Rothman, "Altered cytokine responses of dengue-specific CD4 ${ }^{+} \mathrm{T}$ cells to heterologous serotypes," Journal of Immunology, vol. 175, no. 4, pp. 2676-2683, 2005.

[4] J. Mongkolsapaya, W. Dejnirattisai, X. N. Xu et al., "Original antigenic sin and apoptosis in the pathogenesis of dengue hemorrhagic fever," Nature Medicine, vol. 9, no. 7, pp. 921-927, 2003.

[5] J. Mongkolsapaya, T. Duangchinda, W. Dejnirattisai et al., "T cell responses in dengue hemorrhagic fever: are cross-reactive T cells suboptimal?" Journal of Immunology, vol. 176, no. 6, pp. 3821-3829, 2006.
[6] A. P. Durbin and S. S. Whitehead, "Dengue vaccine candidates in development," Current Topics in Microbiology and Immunology, vol. 338, no. 1, pp. 129-143, 2010.

[7] T. P. Endy, A. Nisalak, S. Chunsuttitwat et al., "Relationship of preexisting dengue virus (DV) neutralizing antibody levels to viremia and severity of disease in a prospective cohort study of DV infection in Thailand," Journal of Infectious Diseases, vol. 189, no. 6, pp. 990-1000, 2004.

[8] D. J. Gubler, "Dengue and dengue hemorrhagic fever," Clinical Microbiology Reviews, vol. 11, no. 3, pp. 480-496, 1998.

[9] S. Wang, R. He, J. Patarapotikul, B. L. Innis, and R. Anderson, "Antibody-enhanced binding of dengue-2 virus to human platelets," Virology, vol. 213, no. 1, pp. 254-257, 1995.

[10] K. I. Yamada, T. Takasaki, M. Nawa, and I. Kurane, "Virus isolation as one of the diagnostic methods for dengue virus infection," Journal of Clinical Virology, vol. 24, no. 3, pp. 203209, 2002.

[11] S. B. Halstead, "Dengue in the Americas and Southeast Asia: do they differ?" Revista Panamericana de Salud Publica, vol. 20, no. 6, pp. 407-415, 2006.

[12] S. B. Halstead, J. E. Scanlon, P. Umpaivit, and S. Udomsakdi, "Dengue and chikungunya virus infection in man in Thailand, 1962-1964. IV. Epidemiologic studies in the Bangkok metropolitan area," American Journal of Tropical Medicine and Hygiene, vol. 18, no. 6, pp. 997-1021, 1969.

[13] A. Balmaseda, S. N. Hammond, L. Perez et al., "Serotypespecific differences in clinical manifestations of dengue," The American Journal of Tropical Medicine and Hygiene, vol. 74, pp. 449-456, 2006.

[14] A. Méndez and G. González, "Dengue haemorrhagic fever in children: ten years of clinical experience," Biomedica, vol. 23, no. 2, pp. 180-193, 2003.

[15] I. Rodriguez-Barraquer, M. T. Cordeiro, C. Braga, W. V. de Souza, E. T. Marques, and D. A. T. Cummings, "From reemergence to hyperendemicity: the natural history of the dengue epidemic in Brazil," PLoS Neglected Tropical Diseases, vol. 5, no. 1, article e935, 2011.

[16] G. P. Kouri, M. G. Guzman, J. R. Bravo, and C. Triana, "Dengue haemorrhagic fever/dengue shock syndrome: lessons from the Cuban epidemic, 1981," Bulletin of the World Health Organization, vol. 67, no. 4, pp. 375-380, 1989.

[17] S. B. Halstead, T. G. Streit, J. G. Lafontant et al., "Haiti: Absence of dengue hemorrhagic fever despite hyperendemic dengue virus transmission," American Journal of Tropical Medicine and Hygiene, vol. 65, no. 3, pp. 180-183, 2001.

[18] H. Loke, D. Bethell, C. X. T. Phuong et al., "Susceptibility to dengue hemorrhagic fever in Vietnam: evidence of an association with variation in the vitamin D receptor and FC $\gamma$ receptor IIA genes," American Journal of Tropical Medicine and Hygiene, vol. 67, no. 1, pp. 102-106, 2002.

[19] B. Acioli-Santos, L. Segat, R. Dhalia et al., "MBL2 Gene polymorphisms protect against development of thrombocytopenia associated with severe dengue phenotype," Human Immunology, vol. 69, no. 2, pp. 122-128, 2008.

[20] A. Sakuntabhai, C. Turbpaiboon, I. Casadémont et al., "A variant in the CD209 promoter is associated with severity of dengue disease," Nature Genetics, vol. 37, no. 5, pp. 507-513, 2005.

[21] R. Soundravally and S. L. Hoti, "Immunopathogenesis of dengue hemorrhagic fever and shock syndrome: role of TAP and HPA gene polymorphism," Human Immunology, vol. 68, no. 12, pp. 973-979, 2007. 
[22] R. Soundravally and S. L. Hoti, "Polymorphisms of the TAP 1 and 2 gene may influence clinical outcome of primary dengue viral infection," Scandinavian Journal of Immunology, vol. 67, no. 6, pp. 618-625, 2008.

[23] S. Vejbaesya, P. Luangtrakool, K. Luangtrakool et al., "TNF and LTA gene, allele, and extended HLA haplotype associations with severe dengue virus infection in ethnic Thais," Journal of Infectious Diseases, vol. 199, no. 10, pp. 1442-1448, 2009.

[24] A. Mathew, I. Kurane, S. Green et al., "Impaired T cell proliferation in acute dengue infection," Journal of Immunology, vol. 162, no. 9, pp. 5609-5615, 1999.

[25] T. H. Nguyen, H. Y. Lei, T. L. Nguyen et al., "Dengue hemorrhagic fever in infants: A Study of Clinical and Cytokine Profiles," Journal of Infectious Diseases, vol. 189, no. 2, pp. 221232, 2004.

[26] K. S. Myint, T. P. Endy, D. Mongkolsirichaikul et al., "Cellular immune activation in children with acute dengue virus infections is modulated by apoptosis," Journal of Infectious Diseases, vol. 194, no. 5, pp. 600-607, 2006.

[27] H. Loke, D. B. Bethell, C. X. T. Phuong et al., "Strong HLA class I-restricted $\mathrm{T}$ cell responses in dengue hemorrhagic fever: a double-edged sword?" Journal of Infectious Diseases, vol. 184, no. 11, pp. 1369-1373, 2001.

[28] H. A. F. Stephens, R. Klaythong, M. Sirikong et al., "HLA-A and -B allele associations with secondary dengue virus infections correlate with disease severity and the infecting viral serotype in ethnic Thais," Tissue Antigens, vol. 60, no. 4, pp. 309-318, 2002.

[29] P. Chiewsilp, R. M. Scott, and N. Bhamarapravati, "Histocompatibility antigens and dengue hemorrhagic fever," American Journal of Tropical Medicine and Hygiene, vol. 30, no. 5, pp. 1100$1105,1981$.

[30] C. LaFleur, J. Granados, G. Vargas-Alarcon et al., "HLA-DR antigen frequencies in Mexican patients with dengue virus infection: HLA-DR4 as a possible genetic resistance factor for dengue hemorrhagic fever," Human Immunology, vol. 63, no. 11, pp. 1039-1044, 2002.

[31] M. L. Paradoa Pérez, Y. Trujillo, and P. Basanta, "Association of dengue hemorrhagic fever with the HLA system," Haematologia, vol. 20, no. 2, pp. 83-87, 1987.

[32] M. T. Cordeiro, U. Braga-Neto, R. M. R. Nogueira, and E. T. A. Marques, "Reliable classifier to differentiate primary and secondary acute dengue infection based on IgG ELISA," PLoS ONE, vol. 4, no. 4, article e4945, 2009.

[33] E. T. A. Marques, R. Maciel Filho, and P. N. August, "Overcoming health inequity: potential benefits of a patient-centered open-source public health infostructure," Cadernos de Saude Publica, vol. 24, no. 3, pp. 547-557, 2008.

[34] A. Igarashi, "Isolation of a Singh's Aedes albopictus cell clone sensitive to dengue and Chikungunya viruses," Journal of General Virology, vol. 40, no. 3, pp. 531-544, 1978.

[35] R. S. Lanciotti, C. H. Calisher, D. J. Gubler, G. J. Chang, and A. V. Vorndam, "Rapid detection and typing of dengue viruses from clinical samples by using reverse transcriptase-polymerase chain reaction," Journal of Clinical Microbiology, vol. 30, no. 3, pp. 545-551, 1992.

[36] OPAS, Dengue y Dengue Hemorrágico en Las Américas: Guías Para su Prevención y Control, Organización Panamericana de la Salud, Washington, DC, USA, 1995.

[37] M. Bunce, C. M. O’Neill, M. C. N. M. Barnardo et al., "Phototyping: comprehensive DNA typing for HLA-A, B, C, DRB1, DRB3, DRB4, DRB5 and DQB1 by PCR with 144 primer mixes utilizing sequence-specific primers (PCR-SSP)," Tissue Antigens, vol. 46, no. 5, pp. 355-367, 1995.

[38] O. Olerup and H. Zetterquist, "HLA-DR typing by PCR amplification with sequence-specific primers (PCR-SSP) in 2 hours: an alternative to serological DR typing in clinical practice including donor-recipient matching in cadaveric transplantation," Tissue Antigens, vol. 39, no. 5, pp. 225-235, 1992.

[39] P. Louzada, N. H. Deghaide, M. B. Araujo et al., "Immunobiology of the human MHC," in Proceedings of the 13th International Histocompatibility Workshop and Conference, J. A. Hansen, Ed., pp. 647-648, 2007.

[40] F. Williams and D. Middleton, "Immunobiology of the human MHC," in Proceedings of the 13th International Histocompatibility Workshop and Conference, J. A. Hansen, Ed., pp. 646-647, 2007.

[41] S. Hatch, T. P. Endy, S. Thomas et al., "Intracellular cytokine production by dengue virus-specific $\mathrm{T}$ cells correlates with subclinical secondary infection," Journal of Infectious Diseases, vol. 203, no. 9, pp. 1282-1291, 2011.

[42] S. Shresta, J. L. Kyle, H. M. Snider, M. Basavapatna, P. R. Beatty, and E. Harris, "Interferon-dependent immunity is essential for resistance to primary dengue virus infection in mice, whereas T- and B-cell-dependent immunity are less critical," Journal of Virology, vol. 78, no. 6, pp. 2701-2710, 2004.

[43] H. H. Lin, S. Ray, S. Tongchusak, E. L. Reinherz, and V. Brusic, "Evaluation of MHC class I peptide binding prediction servers: applications for vaccine research," BMC Immunology, vol. 9, article 8, 2008.

[44] A. B. SABIN, "Research on dengue during World War II," The American Journal of Tropical Medicine and Hygiene, vol. 1, no. 1, pp. 30-50, 1952.

[45] H. Y. Lei, T. M. Yeh, H. S. Liu, Y. S. Lin, S. H. Chen, and C. C. Liu, "Immunopathogenesis of dengue virus infection," Journal of Biomedical Science, vol. 8, no. 5, pp. 377-388, 2001.

[46] I. Zivna, S. Green, D. W. Vaughn et al., “T cell responses to an HLA-B $* 07$-restricted epitope on the dengue NS3 protein correlate with disease severity," Journal of Immunology, vol. 168, no. 11, pp. 5959-5965, 2002.

[47] C. P. Simmons, T. Dong, N. V. Chau et al., "Early T-cell responses to dengue virus epitopes in Vietnamese adults with secondary dengue virus infections," Journal of Virology, vol. 79, no. 9, pp. 5665-5675, 2005.

[48] T. Duangchinda, W. Dejnirattisai, S. Vasanawathana et al., "Immunodominant T-cell responses to dengue virus NS3 are associated with DHF," Proceedings of the National Academy of Sciences of the United States of America, vol. 107, no. 39, pp. 16922-16927, 2010.

[49] S. Green, S. Pichyangkul, D. W. Vaughn et al., "Early CD69 expression on peripheral blood lymphocytes from children with dengue hemorrhagic fever," Journal of Infectious Diseases, vol. 180, no. 5, pp. 1429-1435, 1999.

[50] H. A. F. Stephens, "HLA and other gene associations with dengue disease severity," Current Topics in Microbiology and Immunology, vol. 338, no. 1, pp. 99-114, 2010.

[51] A. M. Khan, O. Miotto, E. J. M. Nascimento et al., "Conservation and variability of dengue virus proteins: implications for vaccine design," PLoS Neglected Tropical Diseases, vol. 2, no. 8, article e272, 2008.

[52] J. Sloan-Lancaster and P. M. Allen, "Altered peptide ligandinduced partial $\mathrm{T}$ cell activation: molecular mechanisms and role in T cell biology," Annual Review of Immunology, vol. 14, pp. 1-27, 1996. 
[53] J. R. Polizel, D. Bueno, J. E. L. Visentainer et al., "Association of human leukocyte antigen DQ1 and dengue fever in a white Southern Brazilian population," Memorias do Instituto Oswaldo Cruz, vol. 99, no. 6, pp. 559-562, 2004.

[54] B. Sierra, R. Alegre, A. B. Pérez et al., "HLA-A, -B, -C, and DRB1 allele frequencies in Cuban individuals with antecedents of dengue 2 disease: advantages of the Cuban population for HLA studies of dengue virus infection," Human Immunology, vol. 68, no. 6, pp. 531-540, 2007.

[55] T. P. Nguyen, M. Kikuchi, T. Q. Vu et al., "Protective and enhancing HLA alleles, HLA-DRB1*0901 and HLA-A*24, for severe forms of dengue virus infection, dengue hemorrhagic fever and dengue shock syndrome," PLoS Neglected Tropical Diseases, vol. 2, no. 10, article e304, 2008.

[56] G. N. Malavige, T. Rostron, L. T. Rohanachandra et al., "HLA class I and class II associations in Dengue viral infections in a Sri Lankan population," PLoS ONE, vol. 6, no. 6, article e20581, 2011. 


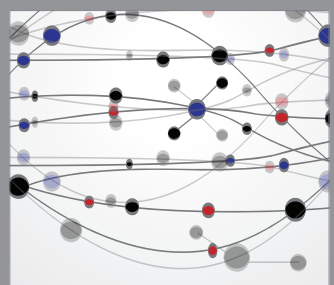

The Scientific World Journal
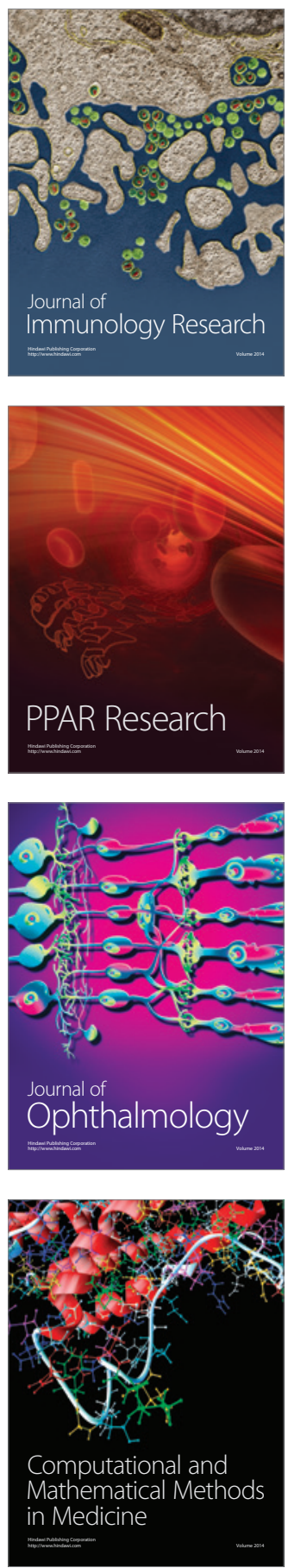

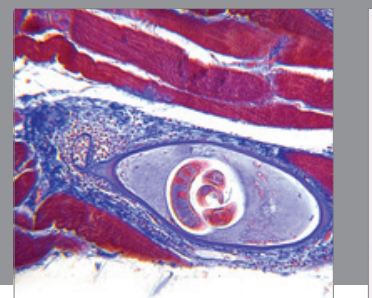

Gastroenterology

Research and Practice
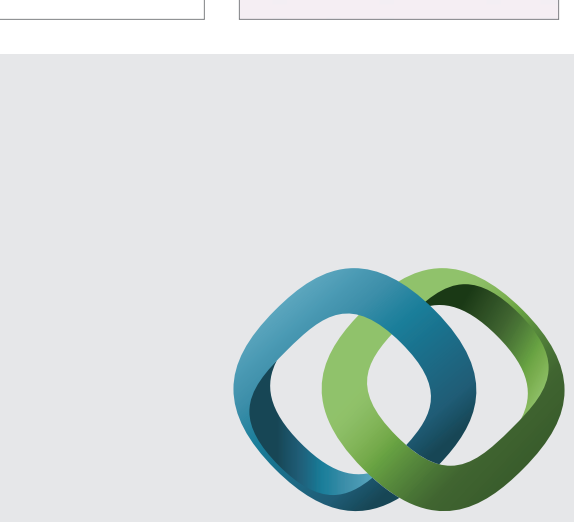

\section{Hindawi}

Submit your manuscripts at

http://www.hindawi.com
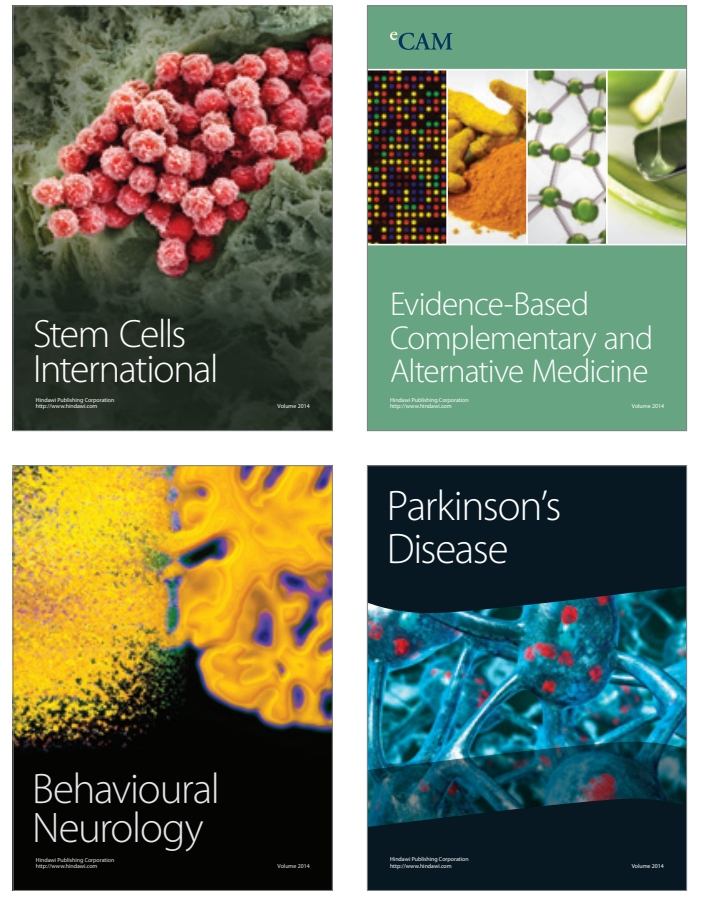
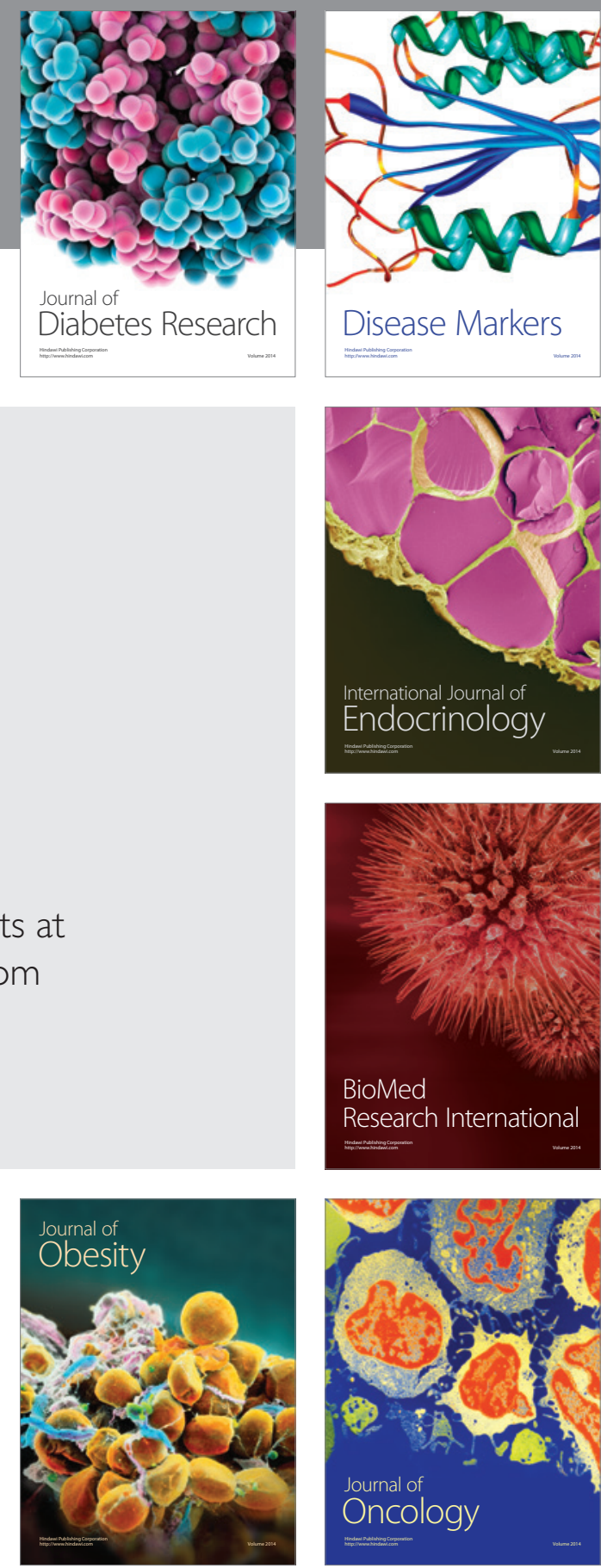

Disease Markers
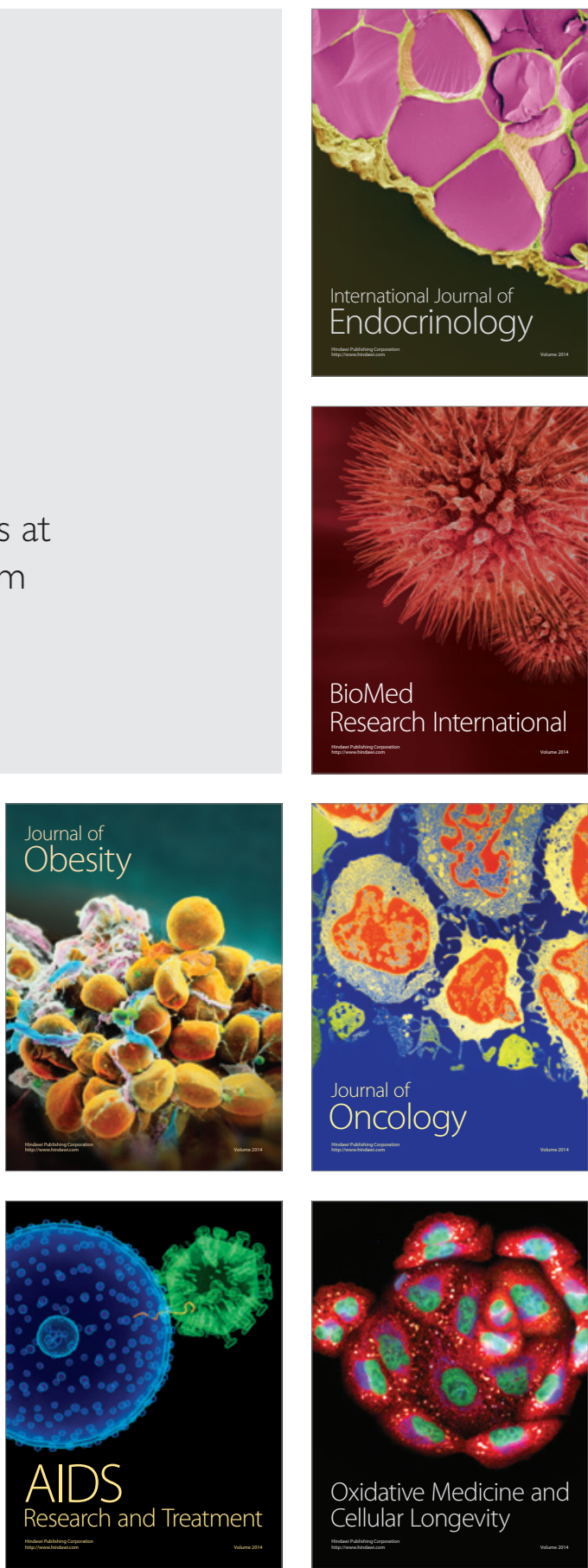